\title{
NON-RIGID IMAGE TRANSFORMATION FOR ASSESSING CHANGES IN FLUORESCENCE IMAGING DATA OF MOLECULAR ACTIVITY IN TIME- DEPENDENT GEOMETRIES
}

\author{
Kostas Marias ${ }^{1}$, Jorge Ripoll ${ }^{2}$, Vasilis Ntziachristos ${ }^{3}$, Stelios Orphanoudakis ${ }^{1,4}$ \\ ${ }^{1}$ Institute of computer Science and ${ }^{2}$ Institute of Electronic Structure \& Laser, Foundation for \\ Research and Technology-Hellas (FORTH), ${ }^{3} \mathrm{MGH} /$ Harvard Medical School CNY Charlestown \\ MA, USA, ${ }^{4}$ Department of Computer Science, University of Crete, Heraklion, Hellas
}

\begin{abstract}
This paper presents an image processing framework for assessing molecular activity changes from fluorescent data in time-dependent geometries. The aim of our work is to provide the necessary tools in order to facilitate molecular imaging studies of small animals, where the basic problem is the time dependent geometry due to different measurement sessions or animal movement. Synthetic, fluorescence imaging data of molecular activity inside a mouse are produced numerically by moving the location of the fluorophore distribution and then randomly and non-linearly transforming the image. A method for aligning the temporal data and improving the accuracy of monitoring fluorophore distribution is presented.
\end{abstract}

\section{INTRODUCTION}

Modern advances in molecular imaging have demonstrated the need for interdisciplinary collaboration so that the full potential of this field is exploited. This paper aims to provide a framework for the computer analysis of temporal molecular imaging data of small animals. Our work is centered on fluorescent data measurements, but the methods presented may as well be applied to any other imaging modality such as MRI, PET, SPECT, and CT.

Lately, there have been great advances in the use of light to probe molecular function (see [1] for a review on the subject). This is mainly due to the large number of specific optical probes and their relatively simple transfection and due to the possibility of engineering transgenics. To date, two main techniques have been used for imaging fluorescent probes, Fluorescence Reflectance Imaging (FRI) [2, 3], which consists of imaging the fluorescence (or luminescence) map emitted from the animal, and Fluorescence-mediated Molecular
Tomography (FMT) [4], which produces 3D spatial maps of fluorophore concentration. Lately, a novel geometry for FMT has been developed, quite similar to that traditionally used for FRI [5]. This geometry consists of illuminating the specimen either from the back (transmission measurements) or from the front (reflection measurements) and taking a flat 2D image of the emitted intensity.

One of the main advantages of the optical method is the fact that it is non-invasive. This opens the possibility of studying disease development, gene expression or cell trafficking over a period of time, on the same subject. However, when performing such experiments, the subject is never placed in the exact same position, making extremely difficult the extraction of accurate values of position and/or full width half maximum (FWHM) of the emitted light. In terms of FMT, this means that the reconstructed images are difficult to compare, due to this position uncertainty. To overcome this problem, we present a method that can account for geometrical distortions and compression differences, transforming the images measured at different stages so that they can be quantitatively compared. The proposed method has the potential to be useful in a large variety of applications that deal with growth factor and/or kinetics, such as cell trafficking and cancer studies.

This paper is structured as follows: In section 2 we describe the simulation data-set and the proposed method for aligning the images, while in section 3 we present a set of results before concluding with a discussion in section 4.

\section{METHODS}

In order to align temporal image data, one can experiment with a plethora of available methods used in medical imaging. However, it has been proven that in diagnostic imaging it is not feasible to develop a generic algorithm for aligning temporal data. The main reasons are the time 
dependent geometry of the object we want to image and the physics of the imaging method used. Therefore, temporal image variations of a certain object include rigid or non-rigid displacements due to e.g. changes in compression and placement of the object as well as intensity transformations caused by factors such as variability in imaging parameters.

In this specific application we simulate timedependent positioning and exerted compression on the subject (mouse), that lead to both rigid and non-rigid changes in the shape of the imaged subject. In order to simulate the fluorescence map emitted from the subject we have made use of the diffusion approximation, modeling the mouse as an infinite slab. This approach has been used for example in FMT studies yielding accurate results (see [5]). Since we are interested in studying the positional error introduced when considering timedependent situations, we have considered the case of a constant distribution of fluorophores that moves along the same XY plane (i.e. parallel to the detection plane) at a distance of $0.75 \mathrm{~cm}$ from the surface. Therefore, we have assumed that the intensity profile does not change, i.e we have not modeled inhomogeneities present in a realistic situation.

By observing such data, we have concluded that intensity-based alignment techniques such as optical flow can't guarantee a good result since the displacement of the mouse in the image as well as the change in compression can be significant. For this reason, we used a variation of a method that has been applied to temporal mammogram registration, based on thin-plate spline interpolation [6]. The analogy of this problem to the mammogram registration one is quite obvious; in both cases the image appearance of an object is distorted over time due to changes in compression. However, in this application the intensity profile is assumed constant over time (while changes in X-ray imaging parameters lead to temporal intensity variability). The method used is described in the following sub-sections.

\subsection{Object-specific landmark localization}

The boundary of the mouse image is a very useful feature since it provides information about the difference in compression and placement of the object between sessions. Moreover, since the shape of the mouse does not change significantly, landmarks can be defined in each pair of temporal images. This is done by using similar concepts with the work described in [6]. First, we use mathematical morphology to obtain an 8-connected outline. Then, using triplets of sampled outline points, we fit a parametric spline and calculate the curvature at each point according to equation (1):

$$
k(t)=\frac{x_{t t} \cdot y_{t}-x_{t} \cdot y_{t t}}{x_{t}^{2}+y_{t}^{2}}
$$

where $x_{t}, x_{t t}$ and $y_{t}, y_{t t}$ are the first and second derivatives of $x(t), y(t)$ with respect to $t$ (the curvilinear parameter $t$ describes the outline).
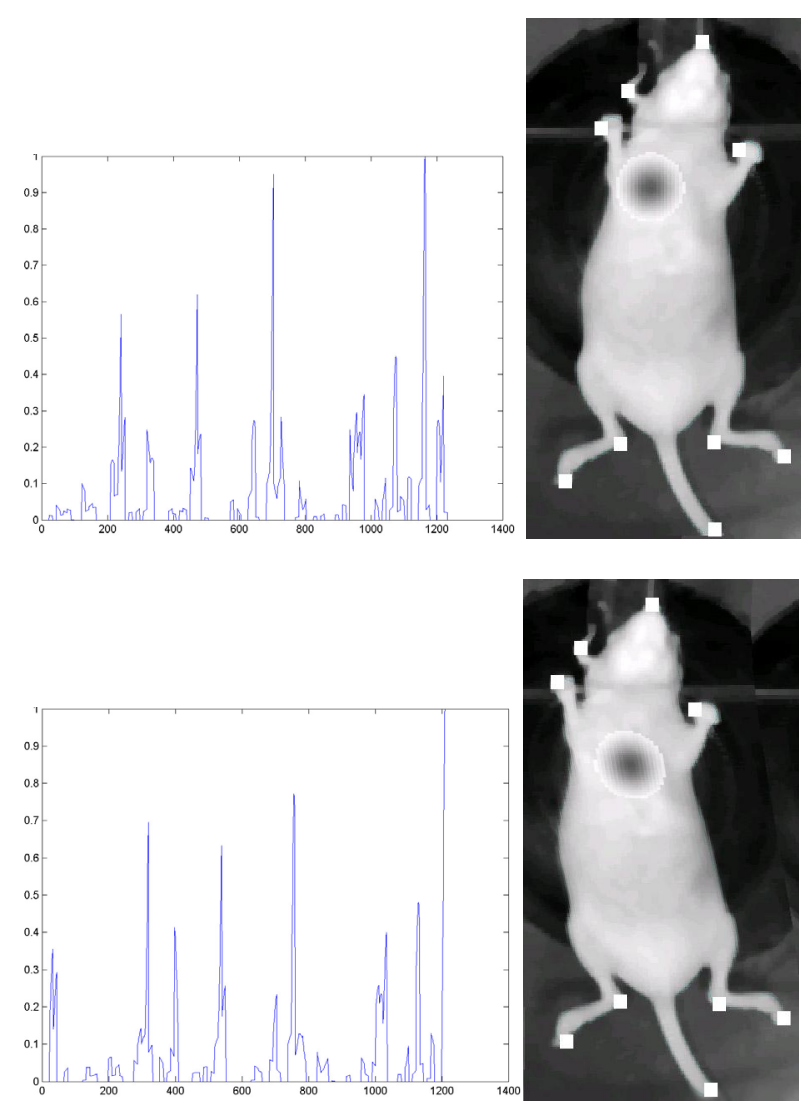

Figure 1. Transformation-invariant landmark detection based on the curvature profiles (shown on the left)

As it is shown in Figure 1, it is possible to detect landmarks on the basis of analyzing the curvature profile. The top right image shows the mouse in its original position while the bottom right one is the result of applying a random non-rigid registration. By comparing the curvature profiles (shown on the left) we can see that the maximum curvature points along the boundaries are transformation invariant. These points can be detected by thresholding the curvature profiles and then using fuzzy clustering to group salient curvature points. The points displayed in Figure 1 (right) are the maximum curvature points of each cluster. It is also noticeable that the curvature profiles are consistent and the small shift is due to the random selection of the starting point along the outline. In this work, we only considered the "positive" curvature profile (moving along the boundary in a clockwise direction, the tangent vector at each positive 
curvature segment, points outside the object). By considering the "negative" value curvature profile, additional maximum curvature points could be detected.

We have experimented with a large variety of possible transformations and have concluded that, in almost all realistic transformation scenarios, the detection of these points is consistent. These points can be the basis of a semi-automatic algorithm for landmark definition. It is important to mention that the robust detection of landmarks is crucial for any feature-based image alignment method. The results indicate that it is possible to further develop this method into a fully automatic system for image alignment and comparison. Once point correspondences are defined, each pair of images is registered as is explained in the next section.

\subsection{Thin-plate spline interpolation}

The basic method that we use for image registration is thin-plate spline interpolation to align temporal mammograms using a set of landmarks from the breast boundary. The calculated interpolating function $f(x, y)$ for the vertical or the horizontal direction is smooth and deforms the image in such a way that the bending energy is minimised, while the landmarks are matched. The bending energy is given by (2):

$$
I_{f}=\iint_{R^{2}}\left(\left(\frac{\partial^{2} f}{\partial x^{2}}\right)^{2}+2 \cdot\left(\frac{\partial^{2} f}{\partial x \partial y}\right)^{2}+\left(\frac{\partial^{2} f}{\partial y^{2}}\right)^{2}\right) d x d y
$$

The implementation details of the method are explained in [7]. This method is efficient at recovering local deformations but special care is needed in the selection of the landmarks. Landmark localization can be based on the curvature analysis described in the above section. To improve registration accuracy, one can sample between pairs of detected transformation-invariant points. In this work, no additional landmarks were used. After the transformation is computed, the template image can be aligned to the target one. The results are presented in the next section.

\section{RESULTS}

Figure 2 shows the application of the proposed non-rigid alignment method in order to correct the time dependent geometry of the mouse simulated here by applying a random non-rigid transformation.
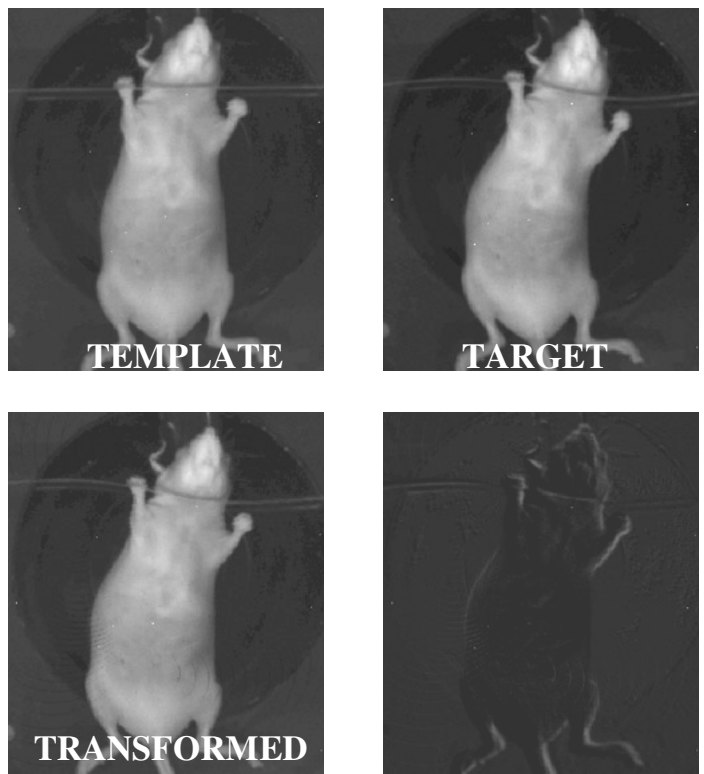

Figure 2. Transformation of the template image (top left) to the target (top right). The transformed image (bottom left) is close to the target, as is verified by the error image (bottom right).

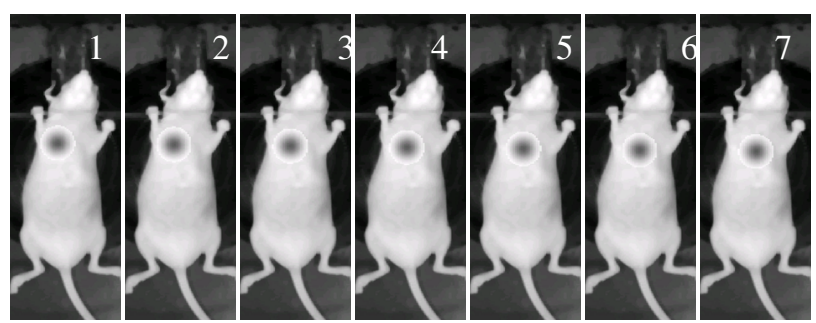

Figure 3. The validation data consists of 7 identical images of the mouse except for the distribution of fluorophores that is simulated to propagate inside the mouse within a plane parallel to the detection plane at $0.75 \mathrm{~cm}$ from the surface.

Figure 3 shows the ground truth data used for a validation experiment. Using only one image of the mouse we simulate the propagation of a constant distribution of fluorophores in 7 different instances. Notice that in Figure 3 the geometry of the mouse is unchanged and each location of the fluorophores is used as the ground truth. Then, a random transformation is applied to each image (2-7) in order to simulate the random changes in positioning and compression. This way, we want to validate the accuracy of our method in recovering these random transformations and locating the site of the fluorophores (center of distribution) in each simulation (2-7). This is further explained if Figure 4, where a random transformation is applied to image 7 of Figure 3. The simulated image (7S) is then aligned with image 1 of Figure 3, resulting in the "corrected" image 7C. 


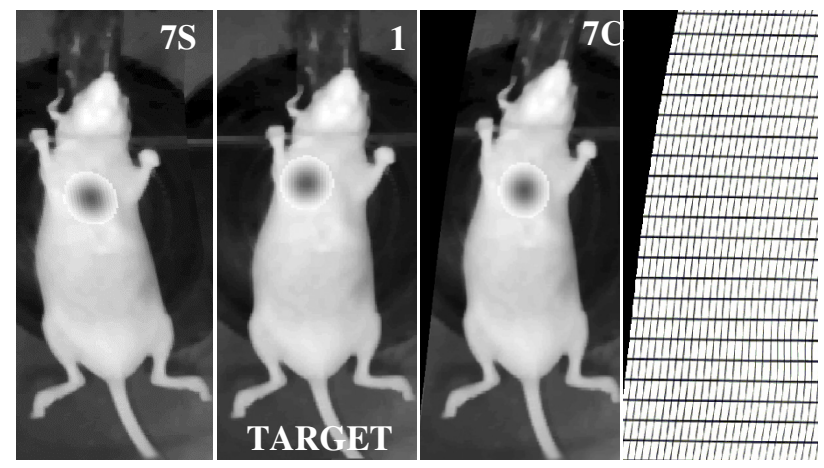

Figure 4 . The simulated image $7 \mathrm{~S}$ is aligned with image 1 of the "ground truth" dataset (see Fig. 3-1) resulting in the corrected image $7 \mathrm{C}$. The last image on the right is the computed transformation grid.

This process is repeated in images 2-6. For validating the accuracy of the method, the location of the center of mass of the fluorophore distribution of the simulated and corrected data-set is compared to the "ground truth" values. The results are plotted in Figure 5. The left graph is the distance of each estimated fluorophore position in the simulated and corrected images from the corresponding "ground truth" position. The right graph shows the distance of all fluorophore center points from the "ground truth" origin point (fluorophore position " 1 " in Figure 3). Specifically, we plot the "ground truth" trajectory of the fluorophore center and compare it to the fluorophore trajectories of the simulated and corrected data-sets. It is obvious that the proposed method can recover most of the image distortions caused by differences in compression and placement of the mouse.

\section{DISCUSSION}

Figure 5 clearly demonstrates that the larger the initial displacement of the region of interest the better is the improvement in localization. The remaining errors are due to landmark localization errors, as well as to a few non realistic transformations produced by randomly distorting the image using commercial image analysis software.

In conclusion, the method presented here may be used in all those instances where flat 2D images are used, such as in Fluorescence Reflectance Imaging and Bioluminescence Imaging and in most PET and SPECT studies. Moreover, its potential application in full 3D imaging is obvious. We believe that the presented method has the potential to play an important role in a large variety of applications that need several imaging sessions, such as cell trafficking, tumor growth factor, and others, considerably improving the resolution and quantization.
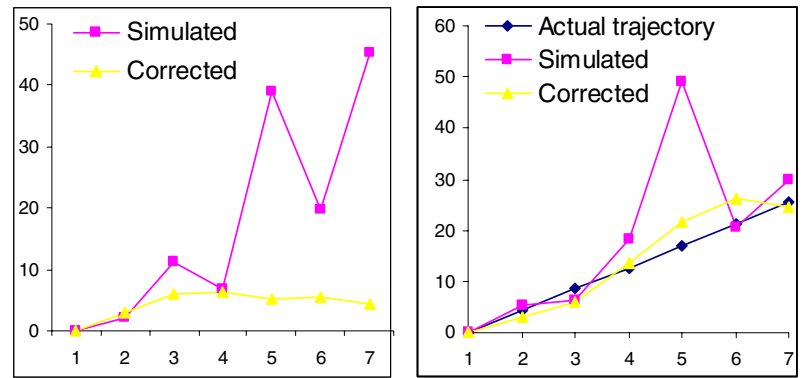

Figure 5. The left graph is a plot of the error distances in pixels (pixel size is $0.037 \mathrm{~cm}$ ) of the center of mass of the fluorophore regions in the simulated and corrected images

(1-7), from the ground truth locations in Figure 3. The right graph shows the "real" trajectory of the fluorophores compared to the trajectories calculated from the simulated and corrected datasets.

\section{ACKNOWLEDGEMENTS}

JR acknowledges support from E.U. project FMRXCT96-0042. VN acknowledges support from NIH grants ROI EB000750-1 and R21-CA91807.

\section{REFERENCES}

[1] R. Weissleder and V. Ntziachristos, "Shedding light onto live molecular targets", Review in Nat. Med., Vol. 9(1), pp. 123-128, 2003.

[2] U. Mahmod, C.H. Tung, A. Bogdanov, R. Weissleder, "Nearinfrared optical imaging of protease activity for tumor detection", Radiology, Vol. 213, pp.866-870, 1999.

[3] C.H. Contag, D. Jenkins, P.R. Contag and R.S. Negrin, "Use of reporter genes for optical measurements of neoplastic disease in vivo", Neoplasia $\mathrm{Vol}$ 2, pp. 41-52, 2000.

[4] V. Ntziachristos, C. Tung, C. Bremer and R. Weissleder, "Fluorescence-mediated tomography resolves protease activity in vivo", Nat. Med., Vol. 8, pp. 575-560, 2002.

[5] E.E. Graves, J. Ripoll, R. Weissleder, and V. Ntziachristos "A sub-millimeter resolution fluorescence molecular imaging system for small animal imaging", Med. Phys. Vol. 30(5), 2003.

[6] K. Marias, C.P. Behrenbruch, M. Brady, S. Parbhoo, A. Seifalian, "Multi-scale landmark selection for improved registration of temporal mammograms", IWDM, Medical Physics Publishing, Toronto, Canada, June 2000.

[7] F. Bookstein, "Principal warps: Thin-plate splines and the decomposition of deformations", IEE Transactions Pattern Analysis and Machine Intelligence, Vol. 11, pp. 567-585, 1989. 